\section{On Sets and Patterns}

\section{Malabika Pramanik}

The study of patterns has long been a focal point in geometric measure theory, combinatorics, and number theory. Equally relevant in discrete and continuous regimes $\left(\mathbb{Z}^{d}\right.$ and $\mathbb{R}^{d}$, though often with startlingly disparate consequences), these problems are often deceptively simple in their formulation and attractive in their visual appeal. Here are a few prototypical examples that have spearheaded the development of the subject:

1. A set occupying a positive proportion of the natural numbers contains arbitrarily long arithmetic progressions. This affirmative answer by Szemerédi to the famous ErdősTurán conjecture is one of the masterpieces of modern mathematics, and a trendsetter in this field. More generally, given a set $A \subseteq \mathbb{Z}^{d}$ with positive density, i.e.,

$$
\begin{aligned}
\limsup _{N \rightarrow \infty} \frac{\#\left(A \cap[-N, N]^{d}\right)}{\#\left([-N, N]^{d}\right)}=d(A)>0, \\
\text { where } \#(A)=\text { cardinality of } A,
\end{aligned}
$$

and any finite configuration $S \subseteq \mathbb{Z}^{d}$ (say a polytope), $A$ contains infinitely many $\mathbb{Z}$-affine copies of $S$. In other words, there are infinitely many pairs $(m, \alpha)$ with $m \in \mathbb{Z}^{d}$ and $\alpha \in \mathbb{Z} \backslash\{0\}$ such that $m+\alpha S \subseteq A$.

2. Contrast this with a problem in the Euclidean setting. A set $S \subset \mathbb{R}$ is said to be universal if every set of positive Lebesgue measure contains an affine copy of $S$. A classical theorem of Steinhaus shows that all finite sets are universal. A famous question of Erdős asks: does there exist an infinite universal set? Despite its superficial analogy with Szemerédi-type questions in $\mathbb{Z}^{d}$, this problem remains unsolved. All results to date merely establish that certain infinite structures are nonuniversal. In particular, we do not know if $\left\{2^{-n}: n \geq 1\right\}$ is universal.

In general, a typical pattern recognition problem goes as follows. "Given a set and a pattern, find out whether the set contains the pattern." In Szemerédi's theorem (example 1 above), the pattern is an arithmetic progression; the set is one of positive density in the integers. In Steinhaus's theorem (example 2 above), the set is of positive Lebesgue measure on the real line; the pattern is an affine copy of a finite set. Needless to say, one would like a definitive

Malabika Pramanik is a professor of mathematics at the University of British Columbia. Her email address is malabika@math. ubc.ca.

For permission to reprint this article, please contact:

reprint-permission@ams.org.

DOI: https://doi.org/10.1090/noti2256 answer to the problem without an exhaustive search, and with limited information about the set.

Stated in this level of generality, the problem lacks mathematical precision. Is the set discrete or in the continuum? How large is it? What additional properties does it have? What does a "pattern" mean? Is a pattern visual or abstract? Does it have to be geometric, analytical, or algebraic? What types of sets are guaranteed to contain certain patterns? Can a set be "rigged" to avoid a pattern? Finding a few different answers to such questions will be the content of my talk at the AMS Spring Sectional Meeting. An important consideration in a precise formulation of the above problem is the notion of size. "Large" could be interpreted in terms of cardinality, nontrivial measure, positive density, or large dimension, notions that we will discuss and compare in the talk. "Patterns" could be arithmetic or geometric progressions, equilateral triangles, parallelograms, or solutions of certain equations. Regardless of the numerous possible variants of such problems, it would seem natural that large sets ought to contain many patterns. Indeed there is a large body of work, drawing upon tools in harmonic analysis, number theory, and additive combinatorics, that support this intuition. Surprisingly, however, there are also results that challenge this intuition, especially when slight variations in size lead to very different conclusions regarding the existence of patterns.

Part of the talk will be devoted to a discussion of results that address issues of existence, abundance, or avoidance of patterns in sets of varying sizes and structural properties. While the goal is to survey the current research in the area, we will also spend some time on the different proof techniques, ranging from classical and modern analysis, to probability, number theory, and ergodic theory, that have been used to tackle such problems.

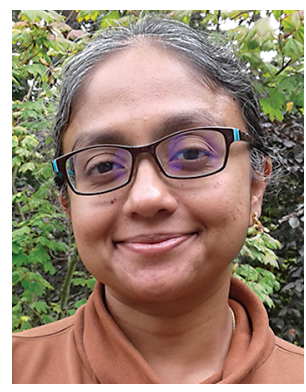

Malabika Pramanik

Credits

Photo of Malabika Pramanik is courtesy of Malabika Pramanik. 